\title{
Release behaviour of amoxicillin from chitosan coated liposomes derived from eggs
}

\author{
M.A.S.K. Menikarachchi ${ }^{1,2}$, K.A.N.P. Katuwavila ${ }^{2,3}$, E.W.M.A. Ekanayake ${ }^{4}$ V. Thevanesam ${ }^{4}$, \\ V. Karunaratne ${ }^{1,3}$ and D.N. Karunaratne ${ }^{1,2^{*}}$ \\ ${ }^{1}$ Department of Chemistry, Faculty of Science, University of Peradeniya, Peradeniya. \\ ${ }^{2}$ Post Graduate Institute of Science, University of Peradeniya, Peradeniya. \\ ${ }^{3}$ Sri Lanka Institute of Nanotechnology, Mahenwatte, Homagama. \\ ${ }^{4}$ Department of Microbiology, Faculty of Medicine, University of Peradeniya, Peradeniya.
}

Revised: 28 September 2015; Accepted: 27 November 2015

\begin{abstract}
A delivery system consisting of chitosan coated liposomes was tested for its ability to deliver drugs over a period of time. Amoxicillin was used as the drug of choice and was entrapped in liposomes made of egg yolk lecithin and coated with chitosan with the aid of Tween 80 and sodium tripolyphosphate. These nanoparticles were monitored for the release of the drug in vitro. The activity of the released amoxicillin was measured against Staphylococcus aureus (NCTC-6571 strain) in BHI broth medium. The percentage of drug released with time was measured by HPLC. The nanoparticles showed sustained release of amoxicillin over a period of 24 hours. Approximately $80 \%$ of the encapsulated drug was released in the first 10 hours. A sufficient drug release to kill the bacteria was obtained in 4 hours and a steady increase in drug concentration was observed up to 8 hours of testing. This study has enabled the model formulation of a sustained release delivery system for amoxicillin in vitro capable of delivering the drug over a period of 8 hours, which may enable drug activity such that the number of times of administration is reduced.
\end{abstract}

Keywords: Amoxicillin, chitosan, drug-carrier system, liposome, sustained release.

\section{INTRODUCTION}

Drug carrier systems are one of the most widely studied areas of research leading to solutions for drug delivery problems such as permeability, bioavailability or solubility of the drugs. Depending on the drug delivery route and the expected properties, various carrier systems were produced in the recent past covering natural and synthetic polymers, liposomes, emulsions and hydrogels. Chitosan, a commonly used polymer for encapsulation is a nontoxic, biodegradable and biocompatible polymer with very interesting biological properties such as permeationenhancing and mucoadhesive properties, anticoagulant and antimicrobial activity. The primary amine groups render special properties that make chitosan very useful in pharmaceutical applications (Berschtetal., 1994). BernkopSchnürch and Dünnhaupt (2012) have reviewed the potential use of chitosan in drug delivery systems in which the cationic nature of chitosan polysaccharides renders properties such as sustained drug release, in situ gelation, transfection and efflux pump inhibitory properties. The carrier function of chitosan depends on its ability to form colloidal particles, which entrap small molecules through a number of mechanisms such as chemical crosslinking, ionic crosslinking, and ionic complexation (BernkopSchnürch \& Dünnhaupt, 2012). In the present study the ionic crosslinking technique has been used.

As a drug delivery system, chitosan has been used in many areas such as oral, ocular, nasal, vaginal, buccal, parenteral and intravesical drug delivery, and also as a vaccine delivery system (Prabaharan \& Mano, 2004). Several researchers have studied the properties of chitosan for delivery of various drugs such as antibiotics [Oxytetracycline (Mi et al., 1999)], anticancer agents [Doxorubicin (Janes et al., 2001)] and pain releivers [dichlofenac sodium (Kumbar et al., 2002)], while Grenha et al. (2005) used chitosan nanoparticles to deliver proteins. 
Liposomal delivery systems are known for their ability to improve the delivery of many drugs. They are particularly useful for delivering water insoluble drugs and also for improving the bioavailability. Drugs entrapped in chitosan nanoparticles have provided enhanced bioavailability. The combination of liposomes with chitosan may enhance the deliverability and bioavailability, and therefore this study focused on using chitosan as a coating agent for liposome formulations due to the affinity of chitosan for cell membranes (Prabaharan \& Mano, 2004).

Amoxicillin is an orally absorbed, broad-spectrum antibiotic, which reaches $\mathrm{C}_{\max }(8 \mu \mathrm{g} / \mathrm{mL})$ about two hours after administration and has an elimination half-life of one hour (Gordon et al., 1972; Kosmidis et al., 1972; Handsfield et al., 1973; Neu, 1974). It has applications in the control of both Gram positive and Gram negative species such as Helicobacter pylori, Enterococcus, Streptococcus and Haemophilus. Prolongation of systemic circulation through encapsulation and slow release of the drug over time are seen as methods for the improvement of drug efficacy. They are useful for maintaining the therapeutic levels of the drug over long periods. It is possible to enable such controlled drug delivery by chemically modifying chitosan, thereby altering the release property (Sahasathian et al., 2007; 2010). Songsurang et al. (2011) have formulated a sustained release tablet in which amoxicillin was coated with ethyl cellulose and formulated with chitosan to impart resistance to acid degradation in the stomach. Amoxicillin encapsulated in chitosan based systems have been reported by several research groups. Angadi et al. (2012) devised a novel system with alginate microbeads coated with chitosan for slow release of amoxicillin. Park and Kim (2009) studied the effect of conjugation versus dispersion of amoxicillin in chitosan hydrogels on release properties, and reported that dispersion of the drug enhanced release.

In the current study, Amoxicillin was entrapped in liposomes and then encapsulated by ionic crosslinking of chitosan with low molecular weight sodium tripolyphosphate (STPP). The method used for the encapsulation is described as ionotropic gelation (Bodmeier et al., 1989), which is one of the significant techniques used for chitosan based delivery systems.

\section{METHODOLOGY}

Phosphatidylcholine was extracted from egg yolk; amoxicillin trihydrate, cholesterol, medium molecular weight chitosan, sodium tripolyphosphate (STPP) and Tween $^{\circledR} 80$ were purchased from Sigma Chemicals
(Sigma Aldrich Inc., USA). All solvents were of reagent grade. Water was always used in distilled, deionised form.

\section{Preparation of amoxicillin-loaded liposome suspension}

To prepare the basic liposomes, phosphatidylcholine (lecithin, egg yolk) and cholesterol were dissolved in chloroform $(15 \mathrm{~mL})$ in the ratio 8:2. The solvent was fully evaporated using a rotary evaporator (Heidolph LABOROTA 4000). Next, deionised water containing amoxicillin $(0.01 \% \mathrm{w} / \mathrm{v}, 10 \mathrm{~mL})$ was poured and the solution was kept under mechanical shaking for $13 / 4 \mathrm{hrs}$ at $45^{\circ} \mathrm{C}$ and left overnight at $4{ }^{\circ} \mathrm{C}$ to obtain a liposome suspension.

\section{Coating process using crosslinked chitosan}

After forming the liposome suspension, the coating process was conducted using chitosan as the coating material. Chitosan was dissolved at a concentration of $0.25 \%(\mathrm{w} / \mathrm{v})$ in a solution with $2 \%(\mathrm{v} / \mathrm{v})$ of acetic acid and $1 \%(\mathrm{w} / \mathrm{v})$ Tween ${ }^{\circledR} 80$. STPP $(0.5 \mathrm{mg} / \mathrm{mL})$ was dissolved in deionised water. The chitosan solution $(25 \mathrm{~mL})$ was flush mixed with an equal volume of STPP solution, and the formation of chitosan-STPP particles began spontaneously via the STPP ionic gelation mechanism. The liposome suspension $(10 \mathrm{~mL})$ was added to the prepared chitosan-STPP solution $(50 \mathrm{~mL})$ with mechanical shaking and continuous sonication (Branson Sonicator; model 2510). The suspension was kept overnight at $4{ }^{\circ} \mathrm{C}$ and centrifuged for $30 \mathrm{~min}$ at $15000 \mathrm{rpm}$ (Sigma 3-30KS, Germany). The supernatant was kept for further analysis of non-bound amoxicillin. The particles were re-suspended in deionised water, frozen with $2 \%(\mathrm{w} / \mathrm{v})$ sucrose and freeze-dried overnight using a freeze-dryer (Edwards K4, Britain).

\section{Determination of encapsulation efficiency and loading capacity}

$($ CLAmox $=$ Chitosan coated liposome with amoxicillin;

$\mathrm{CL}=$ Chitosan coated liposome without amoxicillin)

The drug loading capacity (LC) and loading efficacy (LE) were calculated from two methods using the following equations:

$$
\begin{aligned}
\operatorname{LC}(\%)= & {[(\text { Amox }(\text { total })-\text { Amox }(\text { supernatant })) /} \\
& \text { CLAmox }] \times 100 \\
\operatorname{LE~}(\%)= & {[(\text { Amox }(\text { total })-\text { Amox }(\text { supernatant })) /} \\
& \text { Amox }(\text { total })] \times 100
\end{aligned}
$$




\section{Characterisation of amoxicillin-loaded chitosan particles}

For characterisation purposes, fourier transform infrared (FTIR) spectra of chitosan, CL and CLAmox particles were obtained by using a FTIR spectrometer (IRPrestige21, SHIMADZU). The particle size and zeta potentials of the particles were obtained using a particle size/ zeta potential analyser (Zetasizer Nano ZS, Malvern Instruments Ltd.).

\section{Study of antimicrobial activity of amoxicillin-loaded chitosan particles}

To check the antimicrobial activity, separate tube sets containing $1 \mathrm{~mL}$ each of double strength $3.45 \%$ $(\mathrm{w} / \mathrm{v})$ brain heart infusion (BHI) agar medium were prepared. CLAmox particles (5 $\mathrm{mg}$ ) were suspended in phosphate buffer solution ( $\mathrm{pH} 7.4,5 \mathrm{~mL})$ and inserted into dialysis tubing (cellulose membrane, MW cut off $12000 \mathrm{Da}$ ), sealed the ends and placed in a beaker containing phosphate buffer solution (PBS) (pH 7.4, $75 \mathrm{~mL}$ ). Constant stirring was applied using a magnetic stirrer. After $1 \mathrm{hr}, 1 \mathrm{~mL}$ aliquot from the PBS solution (dialysate) was taken and two fold serially diluted in double strength BHI. To each serial dilution, $10 \mu \mathrm{L}$ drops of $10 \%$ diluted $0.5 \mathrm{Mc}$ Farland units (No. of colonies $1.5 * 10^{6} \mathrm{cfu} / \mathrm{mL}$ ) Staphylococcus aureus (NCTC-6571 strain) culture was added and incubated at $37{ }^{\circ} \mathrm{C}$. The test was repeated for other tube sets at separate time intervals. Turbidity of the solutions was observed after $18-24 \mathrm{hrs}$ incubation. Tubes with less or no turbidity were streaked in blood agar plates for further clarification of the existence of bacteria.

\section{Study of sustained release of amoxicillin}

To check the release properties, nanoparticles $(5 \mathrm{mg})$ were suspended in phosphate buffer solution $(\mathrm{pH} 7.4$, $5 \mathrm{~mL}$ ) in cellulose membrane dialysis tubing (MW cut off $12000 \mathrm{Da}$ ), sealed the ends, and placed in a beaker containing phosphate buffer solution ( $\mathrm{pH} 7.4,75 \mathrm{~mL})$. Constant stirring was applied using a magnetic stirrer. Aliquots $(1 \mathrm{~mL})$ were taken from the beaker and monitored for amoxicillin using $\mathrm{C}_{18}$ HPLC column $(3 \times 150 \mathrm{~mm}$, Agilent Corp.). Mobile phase (50\% acetonitrile/50\% water) was pumped at $0.5 \mathrm{~mL} / \mathrm{min}$ resulting in typical retention times of $2.4 \mathrm{~min}$. Amoxicillin released was detected at $229 \mathrm{~nm}$ and the concentrations were determined from a standard curve plotted using area under the curve for known concentrations.

\section{RESULTS AND DISCUSSION}

\section{Loading capacity and loading efficacy}

Drug loading capacity (LC) and loading efficacy (LE) were calculated using equations 1 and 2, respectively. LC was obtained as $6.08 \%$ and the LE was $51.6 \%$. Grenha et al. (2005) found that the loading capacity for encapsulation of insulin in chitosan microspheres was $22-29 \%$ while the loading efficiency was as high as $65-80 \%$. The observed low value for LE in this study may be due to the method employed for entrapment in liposomes. The liposomes with amoxicillin were formulated using the thin film hydration method, which may not be the optimum method for amoxicillin entrapment.

\section{Characterisation by FTIR}

The FTIR spectra of chitosan, CL and CLAmox particles are shown in Figure 1. In the FTIR spectrum of chitosan, a broad peak around $3200 \mathrm{~cm}^{-1}$ corresponding to N-H and $\mathrm{O}-\mathrm{H}$ stretching vibrations can be observed. In chitosan coated liposomes this peak has shifted from $3200 \mathrm{~cm}^{-1}$ to $3450 \mathrm{~cm}^{-1}$ and appears narrower. The N-H bending peak at $1590 \mathrm{~cm}^{-1}$ in the chitosan spectrum has shifted to $1550 \mathrm{~cm}^{-1}$ in the spectrum of chitosan coated liposomes. The above observations reflect the successful bonding of chitosan to the phospholipid layer of the liposomes. The mechanism of coating involves hydrogen bonding between the polysaccharide and phospholipid head groups (Guo et al., 2003). There is hardly any difference between the spectra of CL and CLAmox since the amoxicillin is encapsulated inside the liposomal bilayer and both particles are coated with chitosan.

\section{Size and zeta potential distribution data}

CL particles had a size range around $37 \mathrm{~nm}$ with a zeta potential of $+30.9 \mathrm{mV}$. Usually liposomes exhibit a negative zeta potential due to the negative charge of the phosphate groups in the membrane. The positive zeta potential value of these particles is due to chitosan, which carries a high positive charge. This again confirms the successful formation of chitosan coating around the phospholipid membrane. In this study, it was possible to synthesise very small, coated liposomes around $37 \mathrm{~nm}$ in size. In some previous studies, thiolated chitosan coated liposomes synthesised for the use in oral peptide delivery were in the size range of $600-700 \mathrm{~nm}$ (Gradauer et al., 2013). In another study where chitosan coated liposomes were used to encapsulate leuprolide, the sizes were 


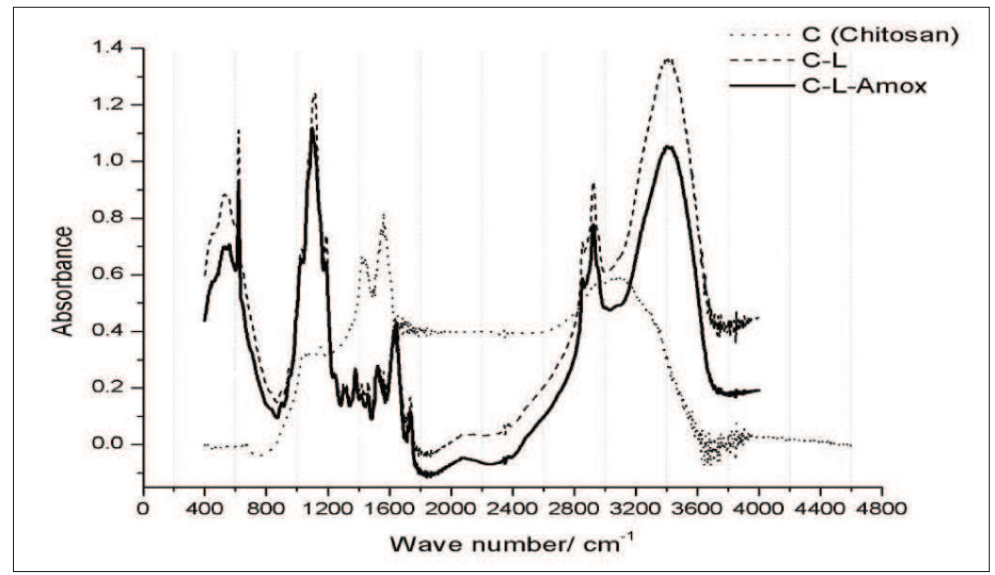

Figure 1: FTIR data for chitosan (C), chitosan coated liposome without amoxicillin (C-L) and chitosan coated liposome with amoxicillin (C-L-Amox)

in the range of $50-100 \mathrm{~nm}$. The sizes increased with increasing concentration of the polymer solution (Guo et al., 2003). When amoxicillin was loaded, the particles (CLAmox) had a size of $18 \mathrm{~nm}$ with a negative zeta potential of $-12.6 \mathrm{mV}$. The negative zeta potential may be due to the negative charge resulting from the carboxyl of amoxicillin. It is notable that no free amoxicillin was found in the encapsulated preparation either by HPLC or antimicrobial activity assays described in the next section where free amoxicillin would have been detected in the first two hours after release.

\section{Antimicrobial study}

In order to evaluate the effect of the carrier on the availability of amoxicillin, the release of free amoxicillin from dialysis tubing was measured over time. Within a period of one and a half hours, all the free amoxicillin dialysed out of the bag. The antimicrobial activity of amoxicillin was tested following the release of drug and the turbidity study indicated the effectiveness of release (Table 1, Figure 2)

Table 1: Activity profile for free amoxicillin released from dialysis membrane

\begin{tabular}{lcccccc}
\hline & & \multicolumn{5}{c}{ Tube } \\
\cline { 3 - 7 } No. & $\begin{array}{c}\text { Time interval/ } \\
\text { hour }\end{array}$ & A & B & C & D & Control \\
\hline 1 & 0.5 & - & - & + & + & + \\
2 & 1.0 & - & - & + & + & + \\
3 & 1.5 & - & - & - & - & + \\
\hline
\end{tabular}

Turbidity observation for each time interval $[\mathrm{ABCD}-\mathrm{BHI}$ with serial dilution of released free amoxicillin; control-BHI without amoxicillin: after incubation at $37{ }^{\circ} \mathrm{C}$ for $18-24$ hours. (-) refers to no turbidity and $(+)$ refers to turbidity].
Table 2: Activity profile for amoxicillin released from CLAmox through dialysis membrane

\begin{tabular}{lcccccc}
\hline & & \multicolumn{5}{c}{ Tube } \\
\cline { 3 - 7 } No. & $\begin{array}{c}\text { Time interval/ } \\
\text { hour }\end{array}$ & A & B & C & D & Control \\
\hline 1 & 1.0 & + & + & + & + & + \\
2 & 2.0 & + & + & + & + & + \\
3 & 3.0 & + & + & + & + & + \\
4 & 4.0 & - & + & + & + & + \\
5 & 5.0 & - & + & + & + & + \\
6 & 6.0 & - & + & + & + & + \\
7 & 6.5 & - & + & + & + & + \\
8 & 7.0 & - & - & + & + & + \\
9 & 7.5 & - & - & + & + & + \\
10 & 8.0 & - & - & - & - & + \\
\hline
\end{tabular}

Turbidity observation for each time interval $[\mathrm{ABCD}-\mathrm{BHI}$ with serial dilution of amoxicillin released from CLAmox; control- BHI without amoxicillin: after incubation at $37^{\circ} \mathrm{C}$ for $18-24$ hours. (-) refers to no turbidity and $(+)$ refers to turbidity].

The same procedure was followed with the CLAmox drug encapsulated system and drug release was monitored over a period of 8 hours. Turbidity observation for amoxicillin released from the CLAmox drug-carrier system over eight hours are shown in Table 2.

The antimicrobial activity of the released amoxicillin was measured against Staphylococcus aureus (NCTC6571 strain) free from beta lactamase activity. The drug encapsulated chitosan particles showed activity of amoxicillin over the period of time evaluated (Table 2). Initial release of amoxicillin was noted after 4 hours and a steady increase in amoxicillin concentration was detected until 8 hours. The dilution series (tube 9A, 10A) at 7.5 and 8 hours respectively, did not show any bacterial colonies 


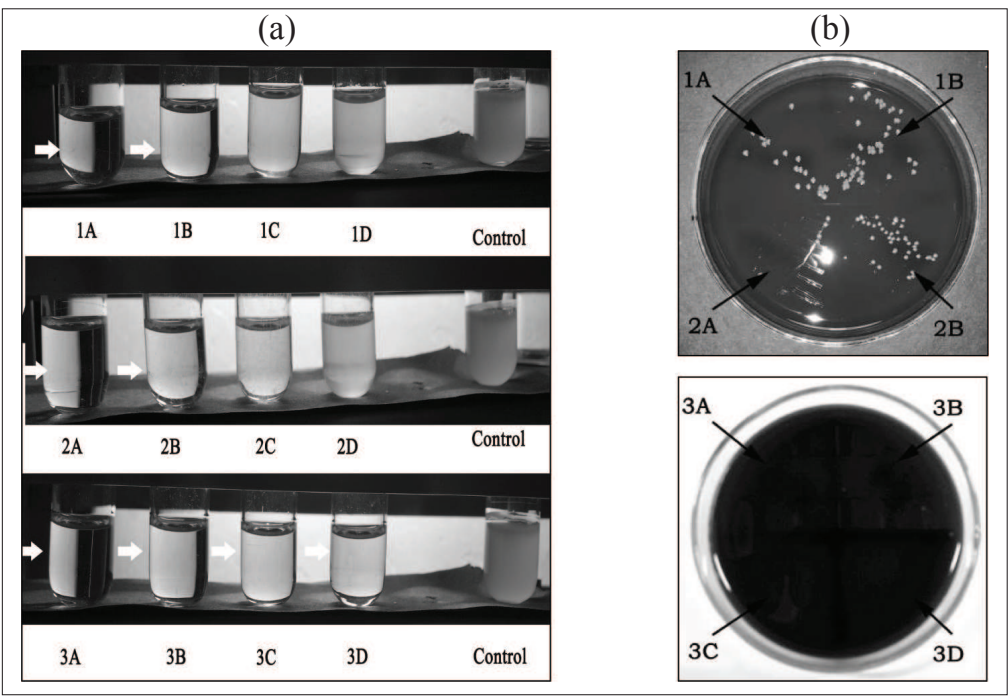

Figure 2: Antibacterial activity of free amoxicillin permeating from dialysis membrane: (a) appearances of tubes containing serial dilution of released free amoxicillin in BHI broth and control (BHI without amoxicillin) after incubation at $37{ }^{\circ} \mathrm{C}$ for $18-24$ hours (tubes with low or no turbidity are indicated with an arrow); (b) streak plates for tubes with low or no turbidity, in blood agar plates.

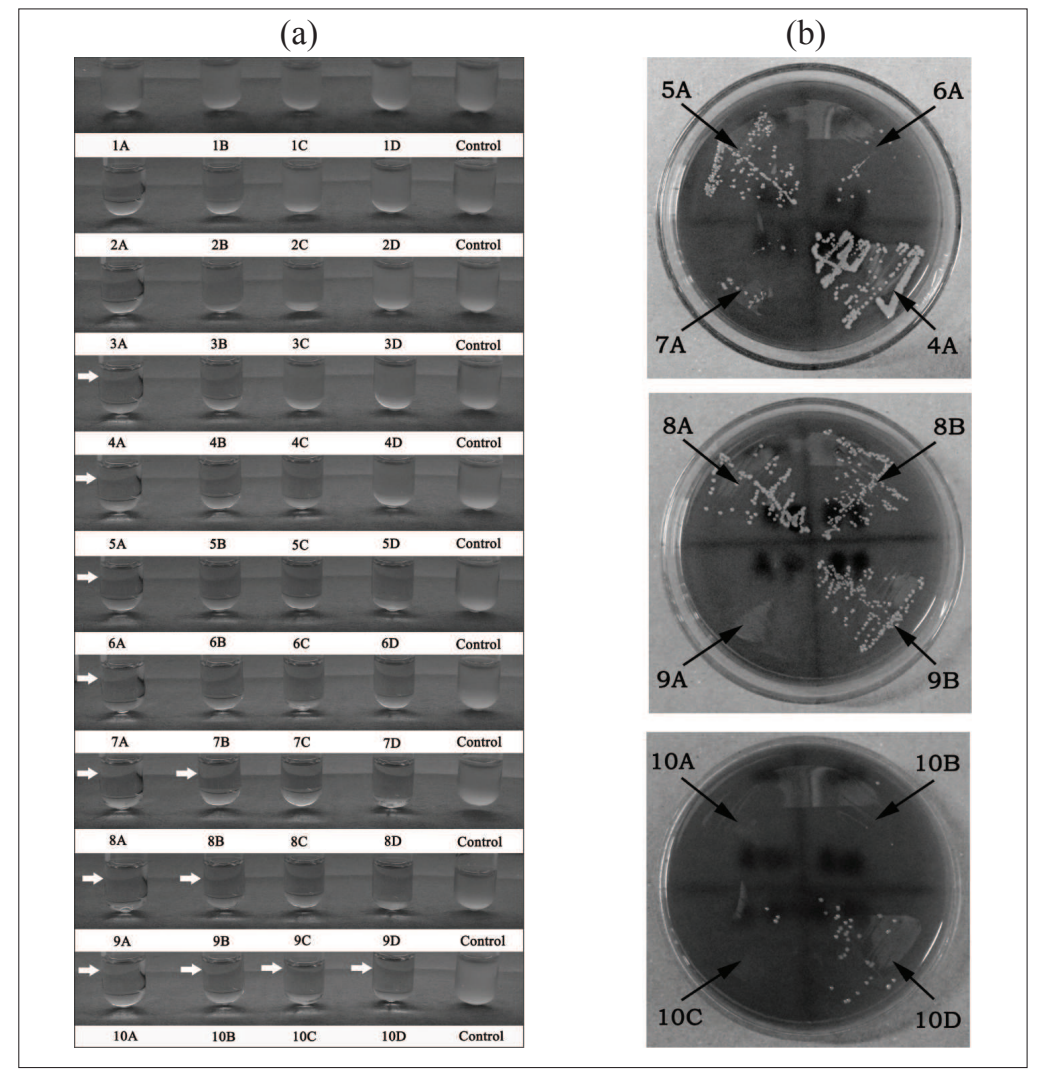

Figure 3: Antibacterial activity of CLAmox permeating from dialysis membrane: (a) appearances of tubes containing serial dilution of amoxicillin (released from CLAmox sample) in BHI broth and control after incubation at $37{ }^{\circ} \mathrm{C}$ for $18-24$ hours (solutions with low or no turbidity are indicated with an arrow); (b) streak plates for tubes with low or no turbidity, in blood agar plates. 


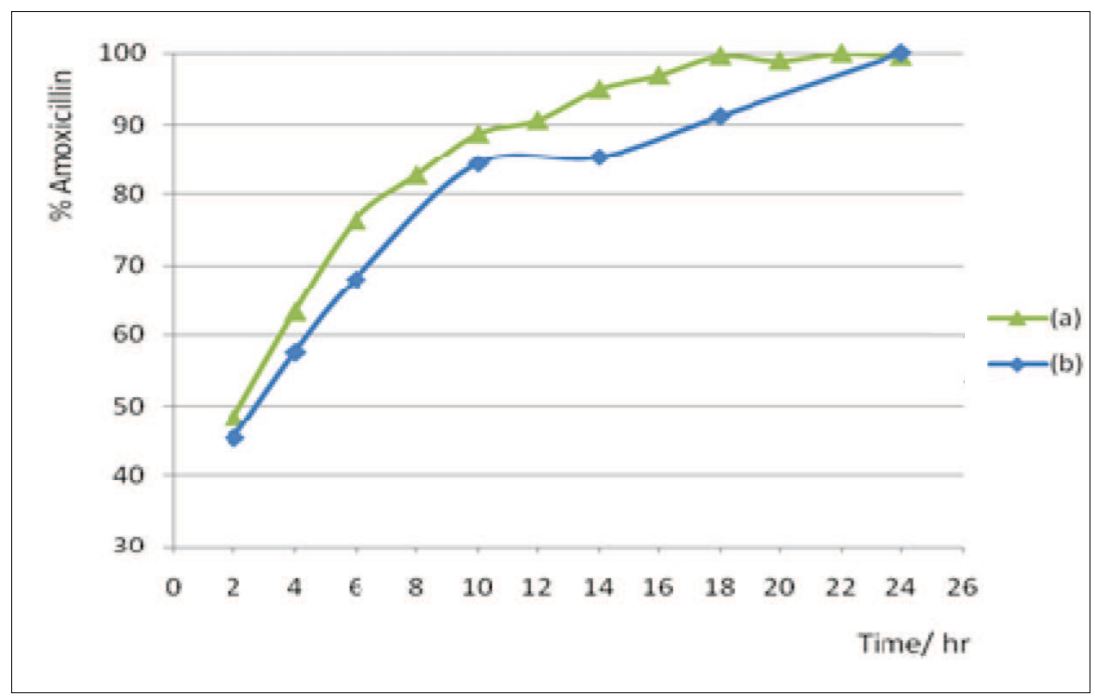

Figure 4: Detection of amoxicillin released at $229 \mathrm{~nm}$ by HPLC

(a) Release behaviour of amoxicillin in liposome; (b) release behaviour of amoxicillin in chitosan coated liposome.

when streaked on blood agar plates (Figure 3). The next dilution (9B and 10B) having decreased concentration (by serial dilution compared to $9 \mathrm{~A}$ and 10A) of released drug also showed that there were no colonies in $10 \mathrm{~B}$ compared to $9 \mathrm{~B}$.

These results show that encapsulating amoxicillin in liposomes followed by coating with chitosan delays the release of the drug. Such a sustained release is of advantage considering that amoxicillin has an elimination half life of 1 hour. Thus the activity of the drug can be prolonged over a longer period compared to the administration of free drug.

\section{Measurement of the amoxicillin released}

The release profile on analysis by HPLC is given in Figure 4. In both cases, the percentage of amoxicillin released in the first six hours amounts to approximately $70 \%$. Liposomal amoxicillin has a slightly greater release [Figure 4(a)] than chitosan coated liposomal amoxicillin [Figure 4(b)]. Thus the coating of liposome with chitosan has resulted in prolonging the release.

\section{CONCLUSION}

Amoxicillin entrapped with chitosan coated liposomal delivery vehicle shows a release profile over 24 hours, which is more beneficial than free amoxicillin release. Inhibition of all the Staphylococcus aureus (NCTC-6571 strain) from the medium was observed 4 hours after the addition of CLAmox with complete inhibition after 8 hours indicating that the period of drug activity can be controlled from the delivery vehicle. The smaller size of the CLAmox particles contribute to the enhancement of activity over other delivery vehicles. Coating the liposomes with chitosan has enabled slower sustained release over the use of uncoated liposomes. Therefore this delivery system can be a potential sustained delivery method for amoxicillin.

\section{Acknowledgement}

Financial assistance from the National Science Foundation of Sri Lanka (Grant RG/2012/NANO/04) is acknowledged.

\section{REFERENCES}

1. Angadi S.C., Manjeshwar L.S. \& Aminabhavi T.M. (2012). Novel composite blend microbeads of sodium alginate coated with chitosan for controlled release of amoxicillin. International Journal of Biological Macromolecules 51(1-2): $45-55$.

DOI: http://dx.doi.org/10.1016/j.ijbiomac.2012.04.018

2. Bernkop-Schnürch A. \& Dünnhaupt S. (2012). Chitosanbased drug delivery systems. European Journal of Pharmaceutics and Biopharmaceutics 81: 463 - 469.

DOI: http://dx.doi.org/10.1016/j.ejpb.2012.04.007

3. Berscht P.C., Nies B., Liebendorfer A. \& Kreuter J. (1994). Incorporation of basic fibroblast growth factor into 
methylpyrrolidinone chitosan fleeces and determination of the in vitro release characteristics. Biomaterials 15(8): $593-600$.

DOI: http://dx.doi.org/10.1016/0142-9612(94)90209-7

4. Bodmeier R., Oh K. \& Pramar Y. (1989). Preparation and evaluation of drug-containing chitosan beads. Drug Development and Industrial Pharmacy 15(9): 1475 - 1494. DOI: http://dx.doi.org/10.3109/03639048909062758

5. Gordon C., Regamey C. \& Kirby W.M. (1972). Comparative clinical pharmacology of amoxicillin and ampicillin administered orally. Antimicrobial Agents and Chemotherapy 1(6): $504-507$.

DOI: http://dx.doi.org/10.1128/AAC.1.6.504

6. Gradauer K. et al. (11 authors) (2013). Liposomes coated with thiolated chitosan enhance oral peptide delivery to rats. Journal of Controlled Release 172: 872 - 878.

DOI: http://dx.doi.org/10.1016/j.jconrel.2013.10.011

7. Grenha A., Seijo B. \& L'opez C.R. (2005). Microencapsulated chitosan nanoparticles for lung protein delivery. European Journal of Pharmaceutical Sciences 25: 427 - 437.

DOI: http://dx.doi.org/10.1016/j.ejps.2005.04.009

8. Guo J., Ping Q., Jiang G., Huang L. \& Tong Y. (2003). Chitosan-coated liposomes: characterization and interaction with leuprolide. International Journal of Pharmaceutics 260: $167-173$.

DOI: http://dx.doi.org/10.1016/S0378-5173(03)00254-0

9. Handsfield H., Clark H., Wallace J.F., Holmes K.K. \& Turck M. (1973). Amoxicillin, a new penicillin antibiotic. Antimicrobial Agents and Chemotherapy 3(2): $262-265$. DOI: http://dx.doi.org/10.1128/AAC.3.2.262

10. Janes K.A., Fresneau M.P., Marazuela A., Fabra A. \& Alonsoa M.J. (2001). Chitosan nanoparticles as delivery systems for doxorubicin. Journal of Controlled Release 73: $255-267$.

DOI: http://dx.doi.org/10.1016/S0168-3659(01)00294-2

11. Kosmidis J., Williams J.D., Andrews J., Goodall J.A. \& Geddes A.M. (1972). Amoxicillin pharmacology, bacteriology and clinical studies. The British Journal of Clinical Practice 26(7): 341 - 346.
12. Kumbar S.G., Kulkarni A.R. \& Aminabhavi T.M. (2002). Crosslinked chitosan microspheres for encapsulation of diclofenac sodium: effect of crosslinking agent. Journal of Microencapsulation 19(2): 173 - 180.

DOI: http://dx.doi.org/10.1080/02652040110065422

13. Mi F.L., Wong T.B., Shyu S.S. \& Chang S.F. (1999). Chitosan microspheres: modification of polymeric chemphysical properties of spray-dried microspheres to control the release of antibiotic drug. Journal of Applied Polymer Science 71: $747-759$.

DOI:http://dx.doi.org/10.1002/(S ICI) 1097 4628(19990131)71:5<747::AID-APP9>3.0.CO;2-6

14. Neu H.C. (1974). Antimicrobial activity and human pharmacology of amoxicillin. The Journal of Infectious Diseases 129: 123 - 131.

DOI: http://dx.doi.org/10.1093/infdis/129.Supplement_2.S123

15. Park J. \& Kim D. (2009). Release behavior of amoxicillin from glycol chitosan superporous hydrogels. Journal of Biomaterials Science Polymer Edition 20(5-6): 853 - 862. DOI: http://dx.doi.org/10.1163/156856209X415855

16. Prabaharan M. \& Mano J.F. (2004). Chitosan-based particles as controlled drug delivery systems. Drug Delivery 12(1): $41-57$. DOI: http://dx.doi.org/10.1080/10717540590889781

17. Sahasathian T., Kerdcholpetch T., Chanweroch A., Praphairaksit N., Suwonjandee N. \& Muangsin N. (2007). Sustained release of amoxicillin from chitosan tablets. Archives of Pharmacal Research 30(4): 526 - 531. DOI: http://dx.doi.org/10.1007/BF02980229

18. Sahasathian T., Praphairaksit N. \& Muangsin N. (2010). Mucoadhesive and floating chitosan-coated alginate beads for the controlled gastric release of amoxicillin. Archives of Pharmacal Research 33(6): 889 - 899. DOI: http://dx.doi.org/10.1007/s12272-010-0612-8

19. Songsurang K., Pakdeebumrung J., Praphairaksit N. \& Muangsin N. (2011). Sustained release of amoxicillin from ethyl cellulose-coated amoxicillin/chitosan-cyclodextrinbased tablets. AAPS PharmSciTech 12(1): $35-45$. DOI: http://dx.doi.org/10.1208/s12249-010-9555-0 